\title{
The Abduction of the Atom: An Exercise in Hypothesizing:
}

\author{
JOSEPH A. NovaK University of Waterloo
}

Keywords: abduction, hypothesis formation, atomic theory, informal logic, scientific revolution, conjecture, affirming the consequent, C.S. Peirce.

Abstract: The paper attempts to schematize, in the form of abductive inferences, the major changes in the developing picture of the atom during the modern period of scientific investigation. The aim of this presentation is to enable students in logic or the philosophy of science to see how a sustained application of abduction might be seen as operative in the development of changing conceptions of the atom, a development which may well be seen as a scientific revolution. The sustained example also illustrates, in pedagogic fashion, the role of images in abduction, the theory-observation distinction, and the analytic-synthetic distinction.

\section{Part I}

Many current textbooks in informal logic and the philosophy of science devote some pages at least to a discussion of the hypothetical method.' This is in marked contrast to, and a genuine advance over, earlier textbooks which generally divided logical reasoning into deductive and inductive, thereby excluding, or only giving marginal consideration to, the method of hypothesis. ${ }^{2}$ However, many of the examples used to show the workings of "hypothesis-making" have a very limited scope and fail to illustrate the complex interplay of moves in the formulation of a major theory. The purpose of this paper is to examine the role of hypothetical reasoning in the case of a series of developments which led to the popular picture of the atom in twentieth century. The advantages of this particular case of hypothetical or abductive reasoning are several. First, the discovery took place over a period of decades-various proposals were formulated by different thinkers in different research areas. This allows one to view the various proposals not simply as logically possible alternatives in the quest of an explanation but more importantly as genuinely distinct contributions which provide historical illustrations of the nuances in the development of an explanation. Second, the process of discovery can be seen as closely tied to an imaginative dimension in the uncovering of a viable explanation of some data. This aspect of the discussion has become more pertinent in the eyes of some current philosophers of science and those working in cognitive science. ${ }^{3}$ Third, the concept of the atom is so familiar and universal and allows such easy entertainment of logical possibilities, that no special historical explanations need be made to get the example off the ground. This example, then, allows for the additional exemplification of the purely logical links within a theory, i.e., the consequences that follow from, or are in conflict with, a theory. 


\section{Part II: Asimov's Summary}

Although some logic texts do employ examples of hypothetical reasoning that bear on significant developments in the history of science, many employ examples that are brief or disengaged from their contexts. In his work, Understanding Physics: The Electron, Proton, and Neutron, Isaac Asimov describes in a very few pages ${ }^{4}$ how our twentieth century conception of the atom came to be. His account is lucid and, although it is somewhat oversimplified- as all popularizations are-it does accurately describe the basic moves involved in the transition from an earlier conception of the atom to the contemporary one. His book, then, serves as a good source for an example that can be analyzed by students to understand better what is occurring in the hypothetical reasoning and other forms of reasoning employed in theory development. Since the paperback text is in print, students have ready access not only to something which provides a rich example, but also to something which will require them to formulate the moves which have occurred in the discovery process. The teacher of logic or philosophy of science can request introductory students read the text and then, as an exercise, "formalize" the steps that took place the process. What follows in Part IV is a proposal to capture at least the chief steps of this process and serve as a model for the exercise.

\section{Part III: Peirce and the Origin of Abduction}

As noted above, several logic texts have already used examples of so-called hypothetical reasoning. This type of reasoning departs from the narrower model of scientific reasoning which was seen as exhausted by either inductive or deductive reasoning or some combination of the two. This narrower model had dominated text-books for a long time. Although there seem to be numerous adherents of this type of reasoning in current circles, one of the great nineteenth century proponents of the reasoning, C.S. Peirce, stood practically alone as the proponent of this structure for hypothetical reasoning. In his collected works, he is found to have said, "abduction must cover all the operations by which theories and conceptions are engendered" (5.590). ${ }^{5}$ He derives the name abduction from what he conjectures was the original Greek in Aristotle. ${ }^{6}$ Abduction is the process whereby an explanatory hypothesis is formed by suggesting what may be the case; it itself cannot be rationally grounded. ${ }^{7}$ While it is itself not rationally grounded, the use of this form of reasoning need not be considered irrational. Although Peirce presents the three types of inference in terms of syllogisms, ${ }^{3}$ he presumably saw this form of reasoning as being capable of assuming other than a syllogistic shape. The present paper will examine the atomic development under consideration by formulating various stages in that development by means of the propositional calculus. 


\section{Part IV: The Analysis of the Development of Atomic Theory}

\section{Stage I}

The first stage is that of Dalton's atom. This is the inherited theory (the foil, if you will) against which the development will take place. Dalton's atom was indivisible and as such it seems that no particles could be emitted from it when exposed to a light source, precisely because it had no parts to emit. (See Model I in the Appendix.) Yet, the theoretical postulation of indivisibility was confronted with the factual evidence of emissions, for when a negatively charged zinc plate was exposed to ultraviolet light, there would be an emission of electrons in a spark. How is this conflict of theory and fact to be explained? There seem to be three options: reject the theory, reject the evidence, or provide an additional theoretical dimension to explain the phenomena. It was this third route that was adopted and that will be considered in the following logical analysis. The letters used will represent the various propositions involved in explaining the initial situation and the subsequent theoretical development: ${ }^{9}$

$\mathrm{H}$ : the atom is indivisible

$\mathrm{C}$ : electrons exist in the interstices of the atoms

$\mathrm{J}$ : electrons are emitted from a material affected by appropriate wavelengths of light

E: electrons are equally emitted by different wavelengths

The initial problem can be represented as:

$$
\mathrm{H} \rightarrow \sim \mathrm{J}
$$

That is, the theory of the atom's indivisibility should exclude any emissions. This is the conflict position which is rectified by the third option of providing an additional theoretical component, namely, that there are interstices between the atoms in which electrons exist and from which, presumably, they could be emitted (C). ${ }^{10}$ This can be represented as $\mathrm{C} \rightarrow \mathrm{J}$. The hypothetical aspect of reasoning which now introduces itself is the (technically invalid) affirmation of the consequent which can be symbolized as:
1). $\mathrm{C} \rightarrow \mathrm{J}$
2). $\mathrm{J}$
3). $\mathrm{C}$

Thus, if the compatibility of $\mathrm{C}$ with $\mathrm{H}$ allows us to assume that $\mathrm{C}$ is a consequent of $\mathrm{H}$, then we can say that $\mathrm{H} \rightarrow \mathrm{C}$. Having supposed that $\mathrm{C} \rightarrow \mathrm{J}$, it seems possible to say that $\mathrm{C} \rightarrow \mathrm{E}$ as well. The fact of the matter is, however, that electrons are not equally emitted by different wavelengths of light." This causes problems for our initial hypothesis $(\mathrm{H})$, as is clear from the following argument:
1). $\mathrm{H} \rightarrow \mathrm{C}$
assumption
2). $\mathrm{C} \rightarrow \mathrm{E}$
assumption
3). $\sim \mathrm{E}$
factual datum
4). $\sim \mathrm{C}$
Modus Tollens 1,2
5). $\sim \mathrm{H}$
Modus Tollens 1,4 
Thus it becomes clear that Dalton's atom cannot explain the emission of electrons even on the supposition that they exist in the interstices between atoms. Hence, one must suppose that the indivisible ballbearing model of the atom (Model I) needs revision.

\section{Stage II}

The second stage is that of Thomson's atom. Given the assumption that negatively charged particles (electrons) existed somehow in association with the atom, and given that they did not exist in the interstices of atoms, the need for a new model arises. Thomson provided this by suggesting the atom was a sphere in which a sufficient number of electrons were embedded to neutralize it. (See Model II in the Appendix.) Thomson's proposal was prompted by some additional considerations. He had to explain first, why the overall charge of the atoms was neutral and second, why only negatively charged particles were emitted. ${ }^{12}$ Clearly, at first blush, the image of a sphere with embedded electrons (similar to a muffin containing chocolate chips) seemed to be one that contained the answers.

The theory allowed the emission of electrons to be easily explained, whether they were emitted as the result of light (earlier designated as $\mathrm{J}$ ) or heat. ${ }^{13}$ The theory has the additional explanatory power of rendering the following two facts intelligible: first, the fact that only negative particles are emitted and not positive ones; second, the fact of ionization, namely, the existence of both (a) positive ions and (b) negative ions. Since the positive component of the atom was too great to be emitted, only electrons would be emitted under the appropriate stimulus. Since the subtraction or addition of electrons would allow for a change in the charge of the atom, the process of ionization could be explained.

Consider the following designation for the elements in the theory process:

I: The atom has a positively charged solid center $\left(I_{1}\right)$ and has embedded electrons $\left(I_{2}\right)$.

P: Positive particles are not emitted.

$\mathrm{K}$ : Ionization occurs for an atom.

$\mathrm{L}$ : There is a loss or gain of electrons.

Thomson's reasoning process, accomplished by means of affirming the consequent, could be represented as the following:
1). $1 \rightarrow P$
assumption
2). $\mathrm{P}$
factual datum
3). I
Affirming the Consequent 1,2

However, it is not this sole instance of affirming the consequent that seems to be operative in Thomson's theorizing. He also seems to have the following at work
1). I $\rightarrow L$
assumption
2). $\mathrm{L} \rightarrow \mathrm{K}$
assumption
3). $I \rightarrow K$
Syllogism 1,2
4). $\mathrm{K}$
factual datum
5). I
Affirming the Consequent 3,4 
It is not always easy to determine which aspects of a theory were used as initial data to be explained (explananda) and which were derived as subsequent consequences predictable by the theory (consequentia) as, for instance, ionization which might seem predictable on a certain model. This only a careful historical study would reveal in each case.

Thomson's picture of the atom as the "raisins in a pound cake" style of atom ${ }^{14}$ can then be called Model II. However, this model, as did Model I, ran into a difficulty and the difficulty resulted in the rejection of the theory. Lenard was confronted by the fact that a stream of particles, rays of a negative charge (cathode rays) could pass through small thicknesses of matter without much scattering. This fact was difficult to reconcile with the "pound cake" model on which there would have to be significant scattering, i.e., the rays would have to wind their ways between the relatively large particles in which electrons were embedded. ${ }^{15}$ Thus, if

\section{$R$ : Cathode rays undergo considerable scattering after passing} through matter.

Lenard supposes, given Model II, that:

$$
\mathrm{I} \rightarrow \mathrm{R} \text {. }
$$

Since, however, the scattering predictable on the basis of Thomson's theory does not occur, the theory falters:
1). $I \rightarrow R$
assumption
2). $\sim R$
factual datum
3). $\sim \mathrm{I}$
Modus Tollens 1,2

\section{Stage III}

Lenard then searches for a new explanatory hypothesis which will account for the lack of scattering. He proposes a picture of the atom in which there is little solid mass, mainly empty space, and in which particles of opposed charge are paired off to yield an overall neutral charge for the atom. (See Model III in the Appendix.) This would allow for the non-scattered emissions of negative charge. ${ }^{16}$ Once again, one is presented with a theory that seems to integrate into a coherent explanatory whole disparate facts needing explanation, a theory that seems to account for not a single explanandum but for multiple explananda. His theory could be expressed as:

$$
\begin{aligned}
& \mathrm{U} \text { : The atom is composed of mainly empty space }\left(\mathrm{U}_{1}\right) \text { and } \\
& \text { constituted of pairs of equal particles of opposing charge }\left(\mathrm{U}_{2}\right) \text {. }
\end{aligned}
$$

Since the failure of rays to scatter is compatible with this hypothesis, one can go on to affirm that $U \rightarrow \sim R$. Hence, the abductive form of Lenard's reasoning can be schematized as the previous cases:
I). $U \rightarrow \sim R$
assumption
2). $\sim \mathrm{R}$ factual datum
3). $U$ Affirming the Consequent 1,2 
Lenard's understanding of the atom's makeup can be envisioned as paired battery terminals and will be designated as Model III. However, there is a problem with this model as well. Given the equal balancing of the positive and negative particles within the atom, it seems logical that the positive particles should be emitted as charged rays as well (anode rays). However, none such were detectable. Let the release be represented propositionally as,

\section{T: Anode rays are released.}

Since this is compatible with the theory $U$, one would then conjecture further, as in the cases above, that $U \rightarrow T$. Of course, there is no need to argue that this conditional cannot itself be derived from some other propositions; quite obviously other intuitions are at work to show that $T$ will be implied by $U$ (e.g., positive particles are not somehow held in place by forces other than those which hold negative ones, positive particles are capable of movement, etc.). Now it becomes clear that the rejection of the theory will follow the same pattern as noted above in the modus tollens type of argument:
1). $\mathrm{U} \rightarrow \mathrm{T}$
assumption
2). $\sim T$
factual observation
3). $\sim \mathrm{U}$
Modus Tollens 1,2

\section{Stage IV}

The fourth stage is that of Rutherford's atom. In looking at Lenard's theory, Rutherford realized that it did account for some of the phenomena, i.e., the atom seemed to be largely empty. He realized that this aspect of the theory was correct, for he had performed an experiment with alpha particles that seemed to substantiate it. He bombarded some metal with these particles and they passed through it-something which would not have been possible on a model of a largely solid atom. ${ }^{17}$ Thus, in rejecting $U$ it is important to remember that $U$ is a compound proposition; the negation of merely one of its components is sufficient to warrant the rejection of the whole. It seems that it is $U_{2}$ that is the objectionable component. Generally, of course, it is possible that both are at fault, but here, on account of the experiment, not only does $U_{1}$ not have to be rejected, but it also seems further confirmed. For it is clear from the propositional calculus that by DeMorgan's law, if

$$
\mathrm{U}=\mathrm{U}_{1} \cdot \mathrm{U}_{2}
$$

then

$$
\sim U=\sim U_{1} \vee \sim U_{2}
$$

As far as the truth value of $U_{2}$, Rutherford did seem to have an additional reason why it was false. As he looked at the fogging on the plate caused by the bombardment, he noticed that it was not as sharp as it would have been if the alpha particles encountered no resistance. Moreover, the resistance seemed to be great enough to cause a considerable deflection of some of the particles. It appeared, then, that there was a sufficiently large component of the atom that was giving rise to this deflection. ${ }^{18}$ Thus, if the atom were composed of binary pairs of 
particles $\left(\mathrm{U}_{2}\right)$ as on the Lenard model, there would be no structure to cause the fogging. Thus, if

F: Alpha bombardment causes diffuse fogging on the photographic plate.

then the following reasoning can take place:
1). $\mathrm{U}_{2} \rightarrow \sim \mathrm{F}$
assumption
2). $F$
factual observation
3). $\sim \mathrm{U}_{2}$
Modus Tollens 1,2

However, this does not leave Rutherford with a new theory as yet. He must develop a positive alternative to the previous ones. His theory will incorporate elements of both the Thomson and Lenard models. From Thomson he will adopt the notion that the atom has a positively charged center $\left(I_{1}\right)$ and from Lenard he will accept the notion that the atom is largely empty space $\left(U_{i}\right)$, although these will be accepted with some modification. The picture of the atom that he draws is that of the nuclear atom: a solid mass surrounded by an electron cloud. Atoms would then be "fluffy balls of foam with a lead pellet at the center of each."1" This is Model IV of the atom. (See Model IV in the Appendix.) His own conception can perhaps be expressed in
$S$ : The atom is a system with a dense nucleus and surrounding electron(s).

Although it is the case that the bombardment experiment can be seen as providing the "evidence" for the theory, i.e.,
1). $S \rightarrow F$ assumption
2). $F$ factual datum
3). $\mathrm{S}$ Affirming the Consequent 1,2

it is possible to view this hypothesis as being a comprehensive one which includes not only elements (albeit sometimes transformed) of the older theories but also some of the data which they tried to explain. That is, not only is it the case that

$$
\mathrm{S} \rightarrow \mathrm{I}_{1} \cdot \mathrm{U}_{1}
$$

but it is also true that

$$
\mathrm{S} \rightarrow(\mathrm{J} \cdot \mathrm{P} \cdot \sim \mathrm{R} \cdot \sim \mathrm{T}) \text {. }
$$

All members of this conjunct could then be seen as together constituting evidence for an affirming of the antecedent $S$. In this way, the comprehensive explanatory power of the Rutherford atom becomes apparent.

\section{Part V: Conclusion}

As mentioned at the start of this piece, the pedagogic advantages of considering this particular development in the history of science are several and benefit both students in logic and students in the philosophy of science. First of all, since the transition from the Dalton atom to the Rutherford atom is not a "quantum leap" (no pun intended), the student is presented with a picture of the development of science that is more detailed than many of the examples provided in logic texts 
(the phlogiston example, the Semmelweis example, etc.). ${ }^{20}$ This can be used to show that the "revolutions" of science are often not as dramatic as portrayed by some authors. This may well have ramifications, of course, regarding the adoption of a realist vs. an instrumentalist view of scientific knowledge-at least one can use the historical illustration to argue the pros or cons of each position. Second, the distinction between experimental observations and theoretical speculations can be used to illustrate the differences which some philosophers have argued exist between theoretical statements and observation statements. Of course, one need not maintain that the distinction is hard and fast; today not many would hold for such a clear division of the two. Indeed, the example can be discussed to illustrate the theoretical aspects of observation statements. Third, the use of images in connection with theorizing becomes obvious in this presentation. Each model contains an image that not only was fuelled by experimental results but which also helped interpret the results and even gave rise to the prediction of other characteristics. Although the use of images in reasoning might be viewed as having little importance by some philosophers, especially those bent on developing eliminative materialist models of mind, other philosophers are now currently revaluing the role of images and the imagination in reasoning. Fourth, the developmental process can contribute, at least indirectly, to an understanding of the distinction between analytic and synthetic types of proposition (another distinction not without its opponents). This is linked to the previous point, for the successive images proposed arise from an analysis of the mere possibilities available, given the evidence. For instance, the question about the release of positive particles in Lenard's model, does not arise out of any empirical data but rather arises out of a logical possibility given the nature of the binary model itself. Similarly, the conjecture that electrons exist in the interstices of the atoms rather than within them results from a juxtaposing of logical options (something is "inside" or "outside"). The independence of analytic propositions from experience might be better illustrated in this way.

In summation, one can say that the development in atomic theory has perhaps been overlooked as an instance of a scientific revolution largely because it is, as many a recent political upheaval on the planet has been, "a quiet revolution". It remains nonetheless-or, perhaps, even on account of this characteristic - a multifaceted exemplification of aspects of reasoning that is pedagogically useful for both students in logic and students in philosophy of science. 


\section{Appendix - Illustrations}
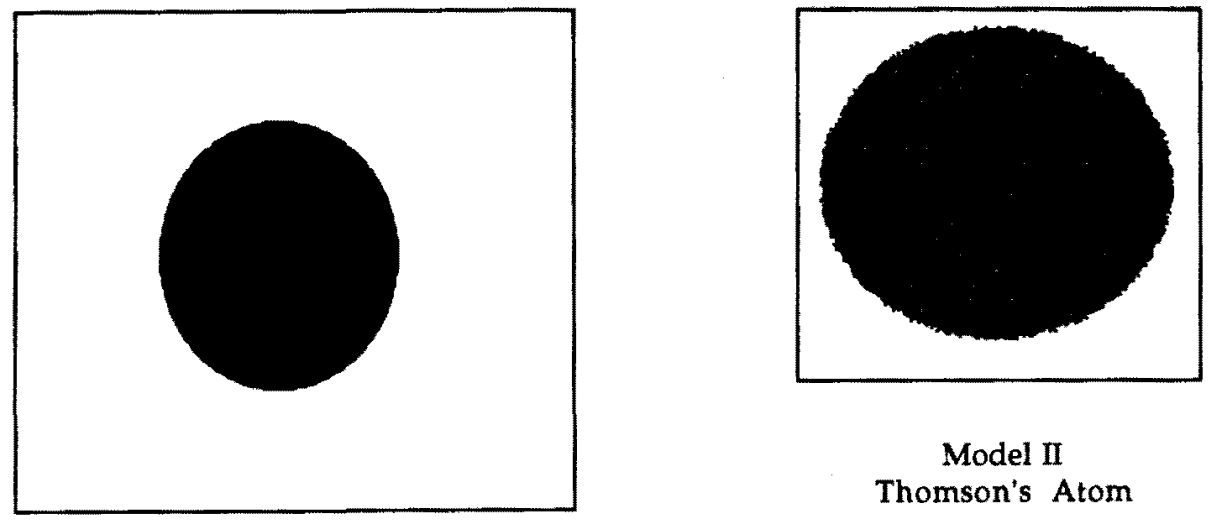

Model II

Thomson's Atom

Model I

Dalton's Atom

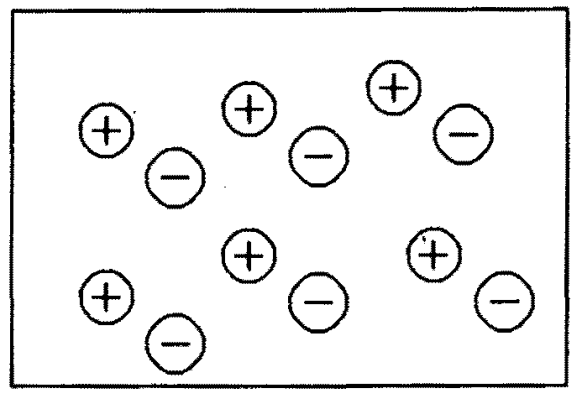

Model III Lenard's Atom

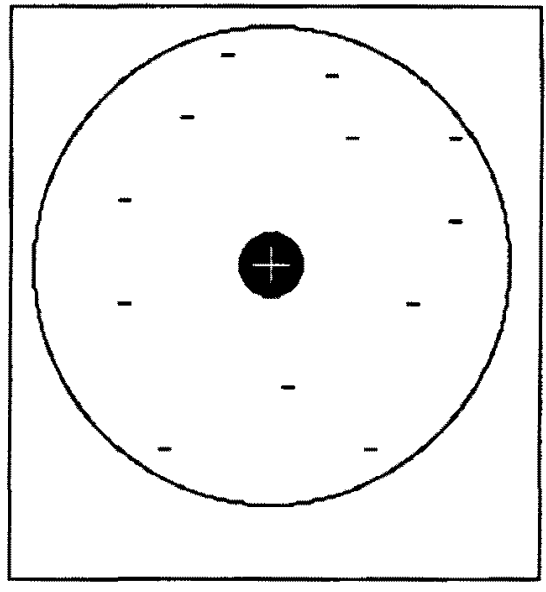

Model IV

Rutherford's Atom 
- My thanks to P. Tenti and F. McCourt, J. van Evra, and R. Holmes of the University of Waterloo for their helpful criticisms. I would also like to thank L. Powers for his work as commentator on this paper.

1 M. Salmon, Introduction to Logic and Critical Thinking (New York: Harcourt Brace Jovanovich, 1989); P. Churchill, Becoming Logical (New York: St. Martin's Press, 1986), c. 11 , pp. 370-408. Often textbooks employ the same examples; of some of those common to the logic texts, R. Giere in his work Understanding Scientific Reasoning (New York: Holt, Rinehart, and Winston, 1979) c. 6, pp. 84-115 seems to be the source.

${ }^{2}$ This method is also sometimes designated the "hypothetico-deductive method." One need only look at the following who omit or marginalize it: R. Purtill, Logic: Argument, Refutation, and Proof (New York: Harper \& Row, 1979); R. B. Angell, in his Reasoning and Logic (New York: Appleton Century Crofts, 1964) holds to the inductive-deductive division in his text, but he does give some consideration to cases of hypothetical reasoning; I. Copi and C. Cohen in their Introduction to Logic (New York, 1990), 8th ed. follow a middle ground-while holding to the basic deduction-induction distinction by giving them separate treatment in Part Two and Part Three respectively of the book, they do include the treatment of hypothesis under Part Three.

${ }^{3}$ Consider for instance A. Goldman who notes this feature in his book Philosophical Applications of Cognitive Science (Oxford: Westview Press, 1993), pp. 51-55.

4 "Chapter Four: Electrons within Atoms", (New York: Signet Books, 1969).

${ }^{5}$ References are to the Collected Papers of Charles Sanders Peirce (Cambridge: Belknap Press of Harvard University Press, 1960).

${ }^{6}$ Section 5.144 "its replacement by a wrong word by his [Aristotle's] first editor, the stupid \{Apellicon\}, has completely altered the sense of the chapter on Abduction." He continues that Aristotle was trying to formulate "that mode of inference which 1 call by the otherwise quite useless name of Abduction - a word which is only employed in logic to translate the [apagoge] of that chapter."

${ }^{7}$ Section 5.172: "It is the only logical operation which introduces any new idea. . . No reason whatever can be given for it . . . and it needs no reason, since it merely offers suggestions."

${ }^{8}$ See sections 2.619-.644; 2.508-.511.

${ }^{9}$ Powers has proposed a more elaborate formulation for the following by utilizing a prior hypothesis $\mathrm{Hl}$ which states that there are no smaller particles involved than the atoms. However, given that the atoms are the ultimate indivisibles on the physical level, the additional hypothesis seems to me unnecessary.

${ }^{10}$ I should note here that the assumptions are twofold: the existence of the electrons in the interstices, as well as their ability to be emitted. These could also be invoked to explain to students the complexity of the reasoning that actually does take place and the frequent enthymematic structure of our reasoning.

${ }^{11}$ Asimov, p. 55: "Philipp Lenard had observed that the energy with which the electrons were ejected depended on the frequency of light, and that light of less than a certain frequency (the threshold value) did not eject electrons. . . . It seems only sensible to consider something always present near the atom, always bound to the atom with a characteristic force, to be part of the atom."

${ }^{12}$ Asimov writes, "The atoms were electrically neutral; if negatively-charged electrons existed about or within the atom, there had to be a positive charge somewhere to neutralize the negative charge of the electrons. If so where was it? Why didn't light ever bring about the ejection of very light positively-charged particles? Why were there only cathode rays, never analogous anode rays?" (p. 56). 
${ }^{13}$ Asimov, p. 57: "Light quanta would jar loose one or more of these electrons, but could scarcely budge the large atom-sphere of positive charge. Again, the heat in a vacuum tube filament would indeed 'boil off" electrons, for as atoms vibrated more strongly with rising temperature ... the electrons would be jarred loose while the atom itself would be essentially unaffected."

${ }^{14}$ This is Asimov's image, p. 57.

${ }^{15}$ Asimov, p.57: "To be sure, the electrons making up the cathode rays were very small and might be pictured as worming their ways between the atoms. If so, they would most likely emerge badly scattered."

${ }^{16}$ Asimov, p. 5: ". . . cathode rays passed through small thicknesses of matter still travelling in an essentially parallel beam, as though they had passed through atoms without much interference."

17 "The stream of alpha particles passed right through the gold leaf as though it were not there and fogged the photographic plate behind it. . . [The gold leaf had] a thickness of 20,000 atoms. ... [This] was strongly in favor of Lenard's notion of an empty atom ...." p. 58.

18 "In fact, Rutherford was able to show that some were deflected more than slightly! About one alpha particle out of every 8000 was deflected through a right angle or even more.... For an alpha particle to be set back on its heels, it must at the very least meet something nearly as massive as itself - something, in short of atom-sized mass."

${ }^{19}$ Asimov, p. 58.

${ }^{20}$ These are found in the texts noted in footnote 1.

JOSEPH A. NOVAK 